\title{
Energetic proton acceleration associated with lo's footprint tail
}

Clark, G.; Mauk, B. H.; Kollmann, P.; Szalay, J. R.; Sulaiman, A. H.; Gershman, D. J.; Saur, J.; Janser, S.; GarciaSage, K.; Greathouse, T.

Total number of authors:

24

Published in:

Geophysical Research Letters

Link to article, DOI:

10.1029/2020GL090839

Publication date:

2020

Document Version

Publisher's PDF, also known as Version of record

Link back to DTU Orbit

Citation (APA):

Clark, G., Mauk, B. H., Kollmann, P., Szalay, J. R., Sulaiman, A. H., Gershman, D. J., Saur, J., Janser, S., GarciaSage, K., Greathouse, T., Paranicas, C., Allegrini, F., Bagenal, F., Bolton, S. J., Connerney, J. E. P. Ebert, R. W., Hospodarsky, G., Haggerty, D., Hue, V., ... Westlake, J. (2020). Energetic proton acceleration associated with lo's footprint tail. Geophysical Research Letters, 47(24), [e2020GL090839]. https://doi.org/10.1029/2020GL090839

\section{General rights}

Copyright and moral rights for the publications made accessible in the public portal are retained by the authors and/or other copyright owners and it is a condition of accessing publications that users recognise and abide by the legal requirements associated with these rights.

- Users may download and print one copy of any publication from the public portal for the purpose of private study or research.

- You may not further distribute the material or use it for any profit-making activity or commercial gain

- You may freely distribute the URL identifying the publication in the public portal 


\section{Geophysical Research Letters}

\author{
RESEARCH LETTER \\ 10.1029/2020GL090839 \\ Key Points: \\ - Juno's likely crossing of Io's Main \\ Alfvén Wing (MAW) during \\ PJ12 reveals evidence of transverse \\ ion acceleration \\ - Observations suggest wave-particle \\ interactions with ion cyclotron \\ waves as the favored acceleration \\ mechanism; however, Alfvén \\ acceleration was not ruled out \\ - Ion conics generated in Io's footprint \\ tail or near the MAW are more \\ intense and energetic than observed \\ in other auroral regions
}

Correspondence to:

G. Clark,

george.clark@jhuapl.edu

Citation:

Clark, G., Mauk, B. H., Kollmann, P., Szalay, J. R., Sulaiman, A. H., Gershman, D. J., et al. (2020). Energetic proton acceleration associated with Io's footprint tail. Geophysical Research Letters, 47, e2020GL090839. https://doi. org/10.1029/2020GL090839

Received 15 SEP 2020 Accepted 3 NOV 2020 Accepted article online 9 NOV 2020

(C)2020. American Geophysical Union. All Rights Reserved.

This article has been contributed to by US Government employees and their work is in the public domain in the USA

\section{Energetic Proton Acceleration Associated With Io's Footprint Tail}

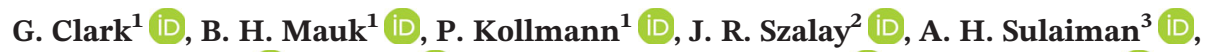

D. J. Gershman ${ }^{4}$ iD, J. Saur ${ }^{5}$ iD, S. Janser ${ }^{5}$, K. Garcia-Sage ${ }^{4}$ iD, T. Greathouse ${ }^{6}$ iD, C. $\operatorname{Paranicas}^{1}$ (iD,

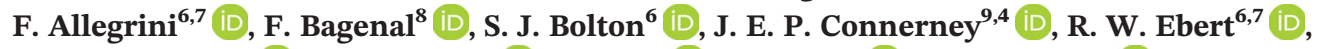

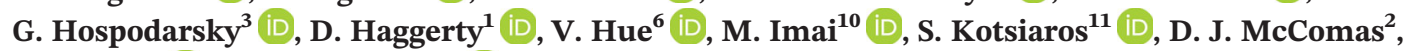
A. Rymer $^{1}$ iD, and J. Westlake ${ }^{1}$ iD

\begin{abstract}
${ }^{1}$ Applied Physics Laboratory, Johns Hopkins University, Laurel, MD, USA, ${ }^{2}$ Department of Astrophysical Sciences, Princeton University, Princeton, NJ, USA, ${ }^{3}$ Department of Physics and Astronomy, University of Iowa, Iowa City, IA, USA, ${ }^{4}$ NASA Goddard Space Flight Center, Greenbelt, MD, USA, ${ }^{5}$ Institute of Geophysics and Meteorology, University of Cologne, Cologne, Germany, ${ }^{6}$ Southwest Research Institute, San Antonio, TX, USA, ${ }^{7}$ Department of Physics and Astronomy, University of Texas at San Antonio, San Antonio, TX, USA, ${ }^{8}$ Laboratory for Atmospheric and Space Physics, University of Colorado, Boulder, CO, USA, ${ }^{9}$ Space Research Corporation, Annapolis, MD, USA, ${ }^{10}$ Department of Electrical Engineering and Information Science, National Institute of Technology (KOSEN), Niihama College, Niihama, Japan, ${ }^{11}$ National Space Institute Measurement and Instrumentation Systems, DTU, Kongens Lyngby, Denmark
\end{abstract}

\begin{abstract}
Observations of energetic charged particles associated with Io's footprint (IFP) tail, and likely within or very near the Main Alfvén Wing, during Juno's 12th perijove (PJ) crossing show evidence of intense proton acceleration by wave-particle heating. Measurements made by Juno/JEDI reveal proton characteristics that include pitch angle distributions concentrated along the upward loss cone, broad energy distributions that span $\sim 50 \mathrm{keV}$ to $1 \mathrm{MeV}$, highly structured temporal/spatial variations in the particle intensities, and energy fluxes as high as $\sim 100 \mathrm{~mW} / \mathrm{m}^{2}$. Simultaneous measurements of the plasma waves and magnetic field suggest the presence of ion cyclotron waves and transverse Alfvénic fluctuations. We interpret the proton observations as upgoing conics likely accelerated via resonant interactions with ion cyclotron waves. These observations represent the first measurements of ion conics associated with moon-magnetosphere interactions, suggesting energetic ion acceleration plays a more important role in the IFP tail region than previously considered.

Plain Language Summary NASA's Juno spacecraft orbits Jupiter's polar region and makes direct measurements of the fields and particles that are responsible for creating Jupiter's powerful auroras. In this article, we present new observations that show intense proton acceleration occurring at altitudes near the auroral emissions created by the interaction between Jupiter's moon Io and the surrounding plasma and magnetic field environment. These unique observations provide clues on how particles are being accelerated and will help constrain particle acceleration theories.
\end{abstract}

\section{Introduction}

Juno's exploration of Jupiter's polar magnetosphere (Bagenal et al., 2017; Connerney, Adriani, et al., 2017) has given prominence to the "far-field" region of the Io-Jupiter interaction with new in situ measurements of Io's footprint (IFP) tail auroral emissions. The far-field interaction specifically refers to the electromagnetic coupling between Io and Jupiter's ionosphere. Decades of remote observations have established that Io generates steady auroral emissions in the radio (Bigg, 1964; Queinnec \& Zarka, 1998; Zarka, 2000), infrared (Connerney et al., 1993), and ultraviolet wavelengths (Clarke et al., 1996; Prangé et al., 1996). Furthermore, more recent HST observations and analyses have characterized its auroral structuring (Bonfond et al., 2008) and correlated brightness changes with Io's centrifugal latitude and system III longitude (Bonfond et al., 2013; Gerard et al., 2006; Hue et al., 2019). Previous flybys of Io from the Voyager and Galileo missions mapped out the local Io-plasma interaction. Plasma and energetic particle (e.g., Belcher et al., 1981; Frank et al., 1996; Gurnett et al., 1996; Williams et al., 1996) and magnetic field (e.g., Acuña et al., 1981; Kivelson et al., 1996) perturbations were not only observed and consistent with theories of Alfvén wing model but also have been discussed in the context of a unipolar inductor model (e.g., Bagenal, 1983; Crary \& Bagenal, 1997; 
Goertz, 1980; Goldreich \& Lynden-Bell, 1969; Gurnett \& Goertz, 1981; Neubauer, 1980; Saur, 2004). Since the Galileo epoch, HST observations (Bonfond et al., 2008, 2009) and modeling efforts (Hess et al., 2010, 2013; Jones \& Su, 2008; Saur et al., 2013) have propelled our understanding of electron acceleration mechanisms generating the Io footprint, for example, highlighting the importance of inertial Alfvén waves. In situ measurements from the Juno mission are revealing new aspects of Io's auroral interaction, which will help further ideas surrounding how particles are accelerated in this region.

Recent analyses of the Juno magnetic field (Gershman et al., 2019), plasma wave (Sulaiman et al., 2020) and the low-energy charged particle data (Szalay et al., 2018; Szalay, Allegrini, et al., 2020; Szalay, Bagenal, et al., 2020) almost universally depict Alfvénic acceleration as a notable, if not dominant, electron acceleration mechanism (Damiano et al., 2019) associated with the IFP tail. More specifically, Gershman et al. (2019) found evidence of transverse magnetic field fluctuations consistent with strong magnetohydrodynamic (MHD) turbulence that can supply $~ 3,000 \mathrm{~mW} / \mathrm{m}^{2}$ of Alfvénic Poynting flux near Io's Main Alfvén Wing (MAW). Similarly, plasma wave observations presented by Sulaiman et al. (2020) show evidence of inertial Alfvén waves, intense ion cyclotron waves, and whistler-mode auroral hiss radiation. Field-aligned lowenergy ( $100 \mathrm{eV} / \mathrm{Q}$ to $100 \mathrm{keV} / \mathrm{Q}$ ) electron beams with broadband energy distributions further support the existence of whistler-mode hiss and the imprints of stochastic particle acceleration via Alfvén waves (Szalay et al., 2018). Finally, a detailed look at the low-energy (10 eV/Q to $46 \mathrm{keV} / \mathrm{Q})$ ion population suggests there is also a significant amount of proton acceleration occurring both at the high latitudes (in similar locations to the electrons) and near the Io torus "boundary"-leading Szalay, Bagenal, et al. (2020) to hypothesize that Alfvén waves generated near Io may be an important acceleration mechanism for the protons as well.

In this work, we are motivated by the aforementioned studies (e.g., Gershman et al., 2019; Sulaiman et al., 2020; Szalay, Allegrini, et al., 2020; Szalay, Bagenal, et al., 2020) to present the higher-energy charged particle observations with a particular focus on the proton data obtained during Juno's 12th perijove (PJ) crossing of the IFP tail in the northern hemisphere. We focus on the proton measurements because the Jupiter Energetic particle Detector Instrument (JEDI) (Mauk, Haggerty, Jaskulek, et al., 2017) observed the most significant ion acceleration event to date, strongly suggesting that the electromagnetic coupling between Io and Jupiter is responsible for energizing protons up to $\sim \mathrm{MeV}$ away from the planet. We compare these data to the magnetic field (Gershman et al., 2019) and plasma wave (Sulaiman et al., 2020) data from the same PJ12 IFP tail crossing near the MAW to better understand the underlying physics governing this unusually intense and unexpected event.

\section{Observations}

\subsection{Juno's Crossing of the IFP Tail}

The data presented here were collected on the inbound leg of PJ12 as Juno crossed the IFP tail in the northern hemisphere between 09:20:35 and 09:20:55 UT on 2018-091 (1 April 2018). Figure 1 is a trajectory schematic comprised of three different representations. Figure 1a illustrates Juno's intersection of a field line that maps to 5.9 Jovian radii $\left(R_{\mathrm{J}}\right.$, where $\left.1 R_{\mathrm{J}}=71,492 \mathrm{~km}\right)$, that is, Io's orbital position, in a cylindrical magnetic dipole coordinate system. Figure $1 \mathrm{~b}$ is a northern polar projection of Jupiter's auroral regions in system III coordinates that encompasses the Io footprint tail (purple curve), Juno's magnetic footprint (orange curve) —calculated using the JRM09 model (Connerney et al., 2018), and the statistical position of the main auroral oval (black trace) derived from Hubble Space Telescope (HST) observations (e.g., Grodent et al., 2003). Juno crossed the IFP tail at an altitude of $0.39 R_{\mathrm{J}}$ and with a longitudinal separation of approximately $1.7^{\circ}$ from Io's MAW spot (e.g., Bonfond et al., 2009) when accounting for the Alfvén wave trajectory bendback between Io and Jupiter's ionosphere. This remains Juno's closest approach to the MAW and potentially a direct crossing (Szalay, Allegrini, et al., 2020). Figure 1c shows Juno ultraviolet spectrometer (UVS) (Gladstone et al., 2017) observations of Jupiter's main auroral oval and the IFP tail approximately 6 min prior to Juno crossing IFP tail. The UVS data are presented in a system III coordinate system with the red trace representing Juno's magnetic footprint.

\subsection{Brief Description of Juno/JEDI}

We focus on observations made by Juno's JEDI. JEDI comprises three sensors (J90, J180, and J270) which measure the energy, angular, and compositional distributions of $>25-\mathrm{keV}$ electrons and $>10-\mathrm{keV}$ ions 

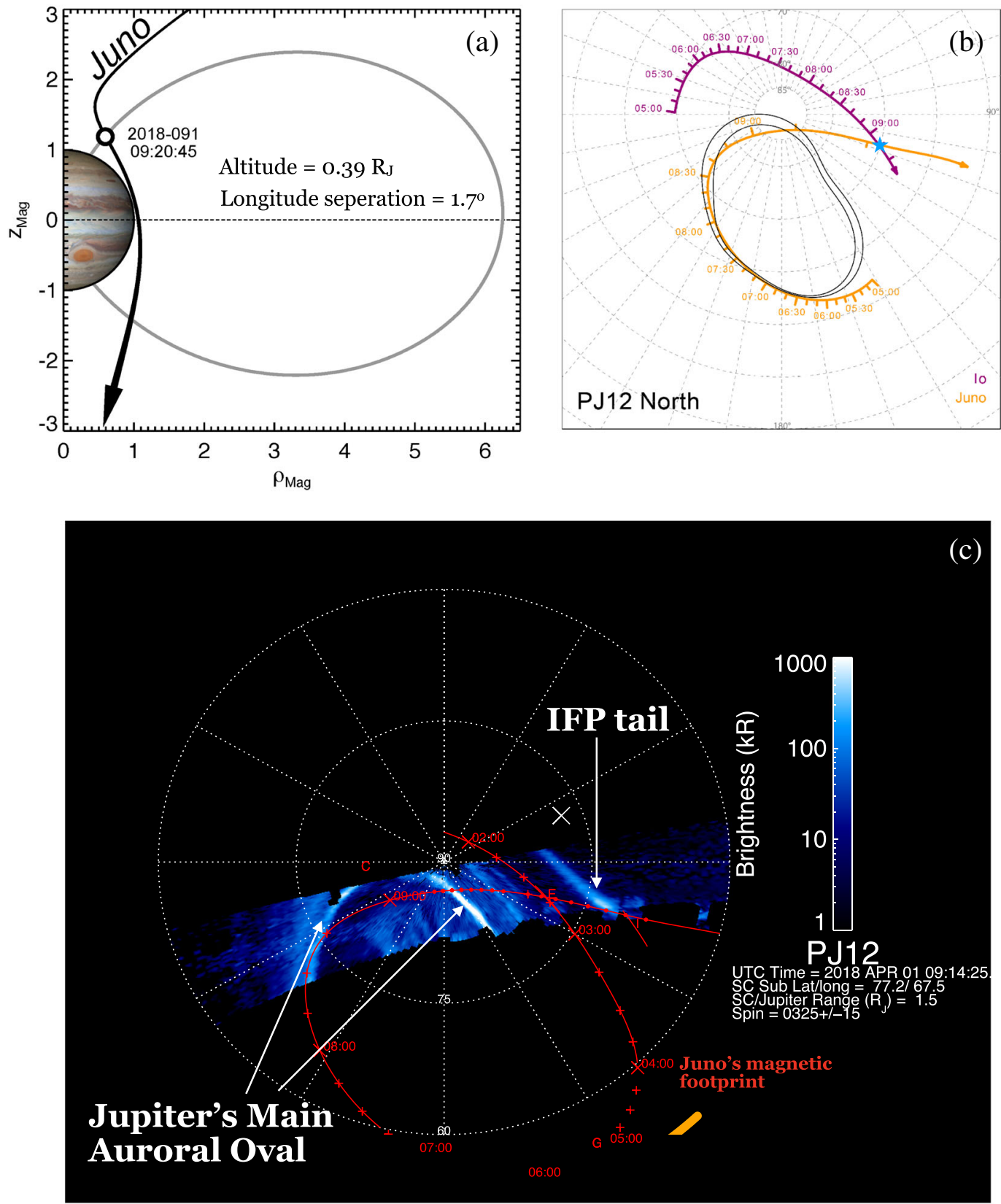

Figure 1. Io footprint tail crossing geometry. (a) Juno's trajectory in cylindrical magnetic coordinates with Io's M-Shell overlaid. (b) Magnetic footprints of Juno (orange curve), Io (purple), and the statistical location of Jupiter's main emission depicted by the bounding black curves. (c) Similar representation as panel (b) but illustrates the ultraviolet brightness observations from Juno/UVS with Juno's magnetic footprint overlaid for reference (red curve). Juno/UVS observations occurred approximately 6 min before Juno crossed the IFP.

(Mauk, Haggerty, Jaskulek, et al., 2017). During this event, the J90 and J270 sensors operated in a high rate mode, thus accumulating time-of-flight by energy rates for $0.25 \mathrm{~s}$ at a cadence of $0.5 \mathrm{~s}$, with no sector averaging. Pitch angle distributions were obtained by combining the JEDI measurements with the measured local magnetic field from Juno/MAG (Connerney, Benn, et al., 2017). The geometric loss cone size at this time is $40^{\circ}$ based on the dipole field approximation and $51^{\circ}$ based on the JRM09 magnetic field model (Connerney et al., 2018). Both methods agree well with the measured loss cone distributions in the ion data. Each solid-state telescope has a full width at half maximum field-of-view (FoV) that is approximately $\sim 17^{\circ} \times 9^{\circ}$ and therefore can resolve the loss cone in this region. The duration of the 
footprint tail crossing is $\sim 20 \mathrm{~s}$, which is shorter than it takes Juno to complete one revolution (Juno spins at approximately two revolutions per minute). This is important because instantaneous look directions and pitch angle averaging between the two sensors can average out fine structure in the IFP tail region. Therefore, we choose to perform all integral moment calculations, that is, characteristic energies and energy fluxes, using a 1-s sampling window over a pitch angle range that contains just the upward moving protons (between $40^{\circ}$ and $90^{\circ}$ ). The integral moment equations are outlined in Mauk et al. (2004) and Clark et al. (2018).

\subsection{Energetic Charged Particle Observations}

Figure 2 presents an overview of the energetic charged particles (Figures 2a-2d) as well as the plasma wave electric field spectral densities (Figure 2e) from Sulaiman et al. (2020) and the transverse magnetic field fluctuations (Figure 2f) from Gershman et al. (2019). Plasma wave and magnetic field measurements were obtained from Juno's Waves (Kurth et al., 2017) and magnetic field (Connerney, Benn, et al., 2017) investigations, respectively. The most prominent feature observed by JEDI is the dramatic proton intensity and pitch angle enhancements (Figures $2 \mathrm{c}$ and $2 \mathrm{~d}$ ) corresponding to the IFP tail. In Figure 2d, protons in the IFP tail are shown to be concentrated along the loss cone (horizontal dashed lines) in the upward direction. There is also evidence of ions streaming upward along the local magnetic field line, but that feature only persists for $\sim 1 \mathrm{~s}$. We do not discuss it further here. The energy-time distribution of the protons (Figure 2c) reveal broad energization ranging from $\sim 50 \mathrm{keV}$ to upward of $\sim 1 \mathrm{MeV}$. During the same time interval, the energetic electrons only show a modest response associated with the IFP tail. Figures $2 \mathrm{a}$ and $2 \mathrm{~b}$ show a slight enhancement in low-energy ( $<60 \mathrm{keV}$ ) electrons and a slight decrease in the very energetic electron environment $(>1 \mathrm{MeV})$, which leaves a signature indicated by the characteristic penetrating charged particle band-a range of residual energies generated by electrons fully penetrating the SSDs-near $160 \mathrm{keV}$ (Mauk et al., 2018). While ions show significant intensities in the upward loss cone, electrons mostly populate the downward loss cone.

Figure 3 shows proton energy spectra and pitch angle distributions for various times associated with IFP tail crossing. The energy distribution of the protons resemble a power law-monotonically decreasing intensities toward increasing particle energy (see Figure 3, left panel). There is no clear evidence of peaked or accelerated Maxwellian-like energy distributions, representative of quasi-static magnetic field-aligned electric fields (Clark, Mauk, Paranicas, et al., 2017; Mauk, Haggerty, Paranicas, et al., 2017; Mauk et al., 2018). In Figure 3, the energy spectra from published Juno/JEDI proton observations are compared (Clark, Mauk, Haggerty, et al., 2017; Mauk et al., 2018). The observations made in the footprint tail suggest that the protons are more efficiently accelerated than in the other auroral regions, which can be seen by the power law curves representing $E^{-2.5}$ and $E^{-3.5}$. Pitch angle distributions for two different times show clear peaks with centroids near $53^{\circ}$ and a full width at the $10 \%$ level of $\sim 30^{\circ}$. Error bars in Figure 3 are determined by estimating the counting uncertainties associated with a Poisson distribution.

In Figure 4, we provide a closer inspection of the energy-time structuring and show the integral moments calculated using a 1-s sampling window over a pitch angle range that contains just the upward moving protons (between $40^{\circ}$ and $90^{\circ}$ ). The energy-time spectrogram in Figure 4a shows discrete stripes that occur somewhat regularly throughout the $\sim 20$-s IFP tail crossing. Similarly, in Figure $4 \mathrm{~b}$, the $100-\mathrm{keV}$ proton intensities are chosen to highlight the variations, which fluctuate by factors of 3-10 on intervals as short as $1 \mathrm{~s}$. Juno provides just a single point measurement and cannot disentangle the temporal/spatial ambiguity; therefore, the 2- to 3-s variations may also be associated with fine spatial structures in the auroral region. It is possible that the variations are a measurement artifact due to the finite angular resolution of JEDI. A crude analysis suggests that a collimated beam of particles can produce an $\sim 2$-s variation in JEDI as a result of Juno's $12^{\circ} / \mathrm{s}$ rotation rate combined with the $\sim 27^{\circ}$ separation between the JEDI telescopes. This sort of temporal variation is observed in the polar cap where electron beams are often narrower than JEDI can resolve (Mauk, Haggerty, Jaskulek, et al., 2017; Paranicas et al., 2018). In this particular event, the variation is likely not an artifact because the measured width of the proton pitch angle distribution is relatively broad, that is, $\sim 30^{\circ}$ (see Figure 3), compared to a single telescope FoV.

The integral moments associated with the IFP tail crossing show energetic protons characteristic energies varying between $\sim 80$ and $400 \mathrm{keV}$ (with a mean $\sim 200 \mathrm{keV}$ ) (Figure 4c) and likewise the proton energy fluxes (Figure $4 \mathrm{~d}$, averaged over pitch angles $40^{\circ}$ to $90^{\circ}$ from $\sim 50 \mathrm{keV}$ to $1 \mathrm{MeV}$ ) to vary between $\sim 10$ and 

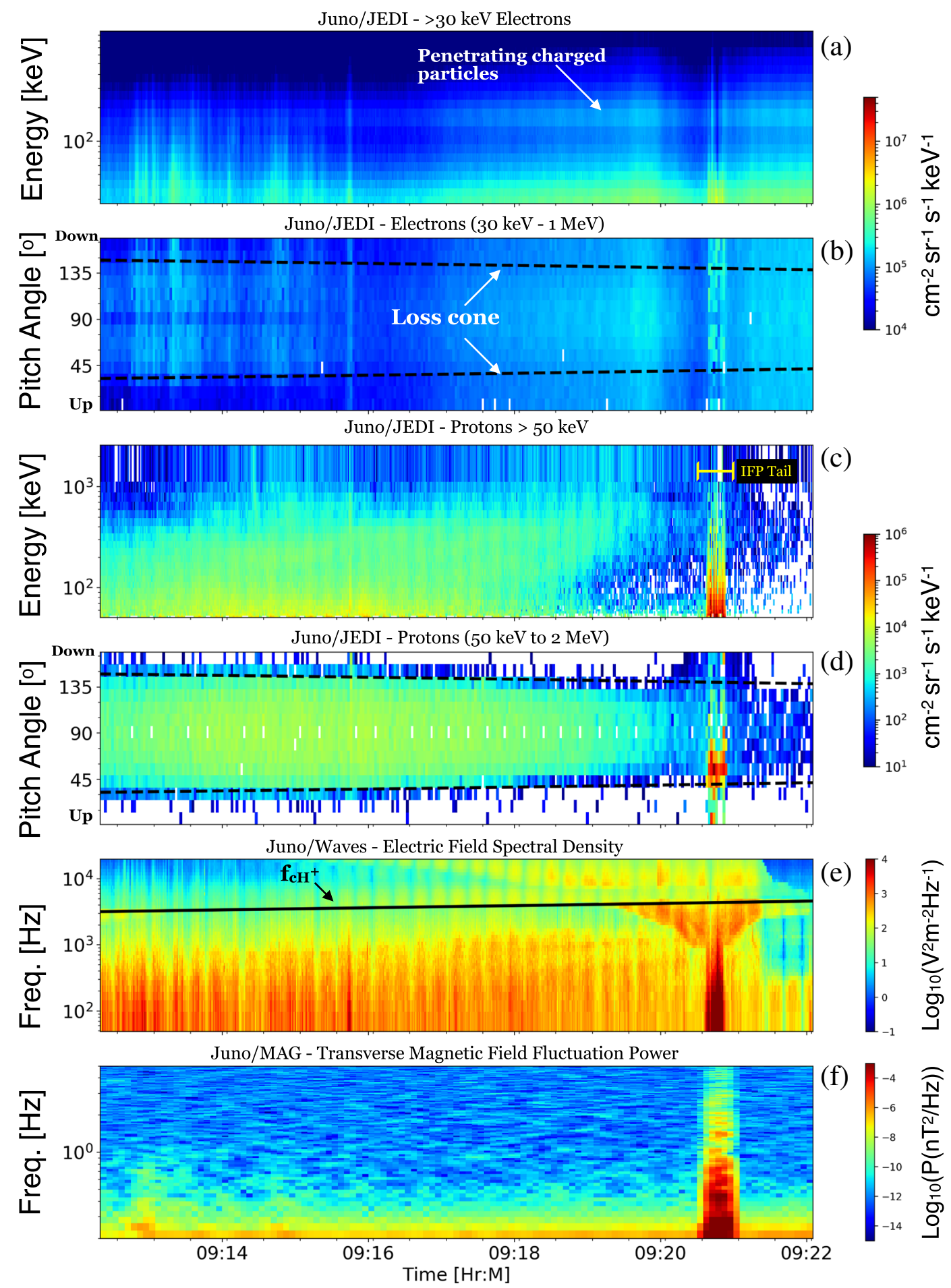

Figure 2. Particles and fields overview of the Juno PJ12 IFP tail crossing. (a-d) The Juno/JEDI observations of the energetic electrons and protons. ( $a$ and b) Energetic electron energy-time and pitch angle-time spectrograms, respectively. (c and d) Energetic proton energy-time and pitch angle-time spectrograms, respectively. (e) Electric field frequency-time spectrogram and (f) magnetic field frequency-time spectrogram. The black dashed lines in (c) and (d) represent the size of the loss cone in degrees using the dipole field approximation. The black solid curve in (e) represents the proton cyclotron frequency derived by Juno/MAG (Sulaiman et al., 2020). 

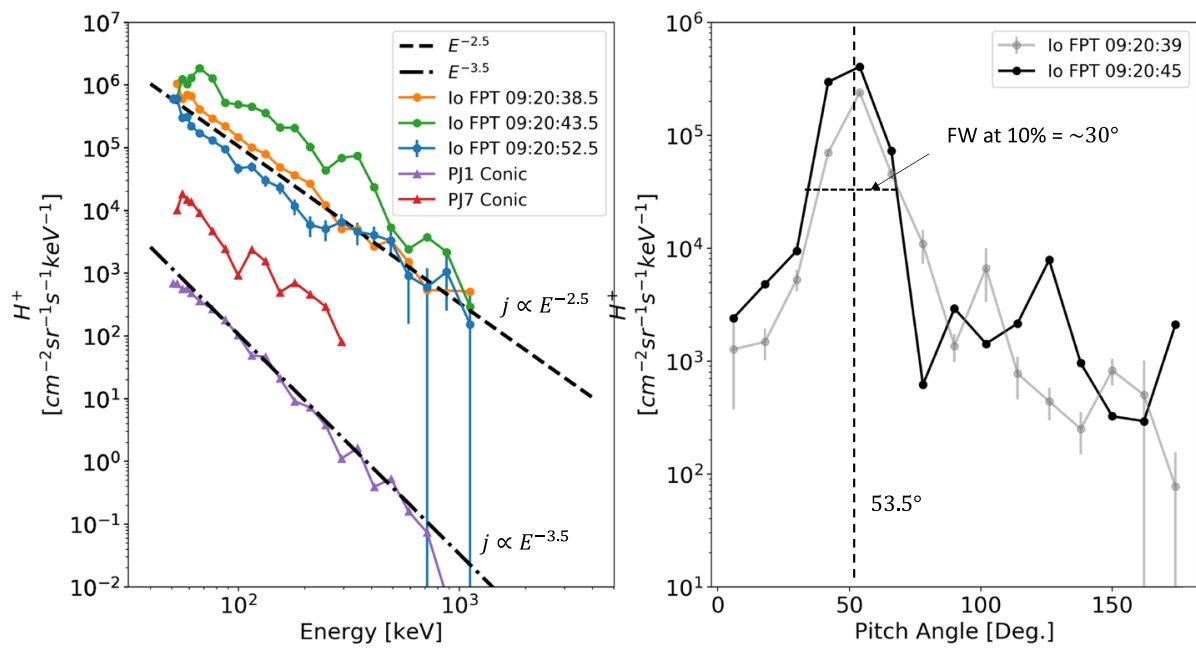

Figure 3. Measured energy spectra (left panel) and pitch angle distributions (right panel). For comparison purposes, energy spectra of proton conic distributions observed during PJ1 (Clark, Mauk, Paranicas, et al., 2017) and PJ7 (Mauk et al., 2018) are also shown as well as power law curves illustrating the different spectral slopes.
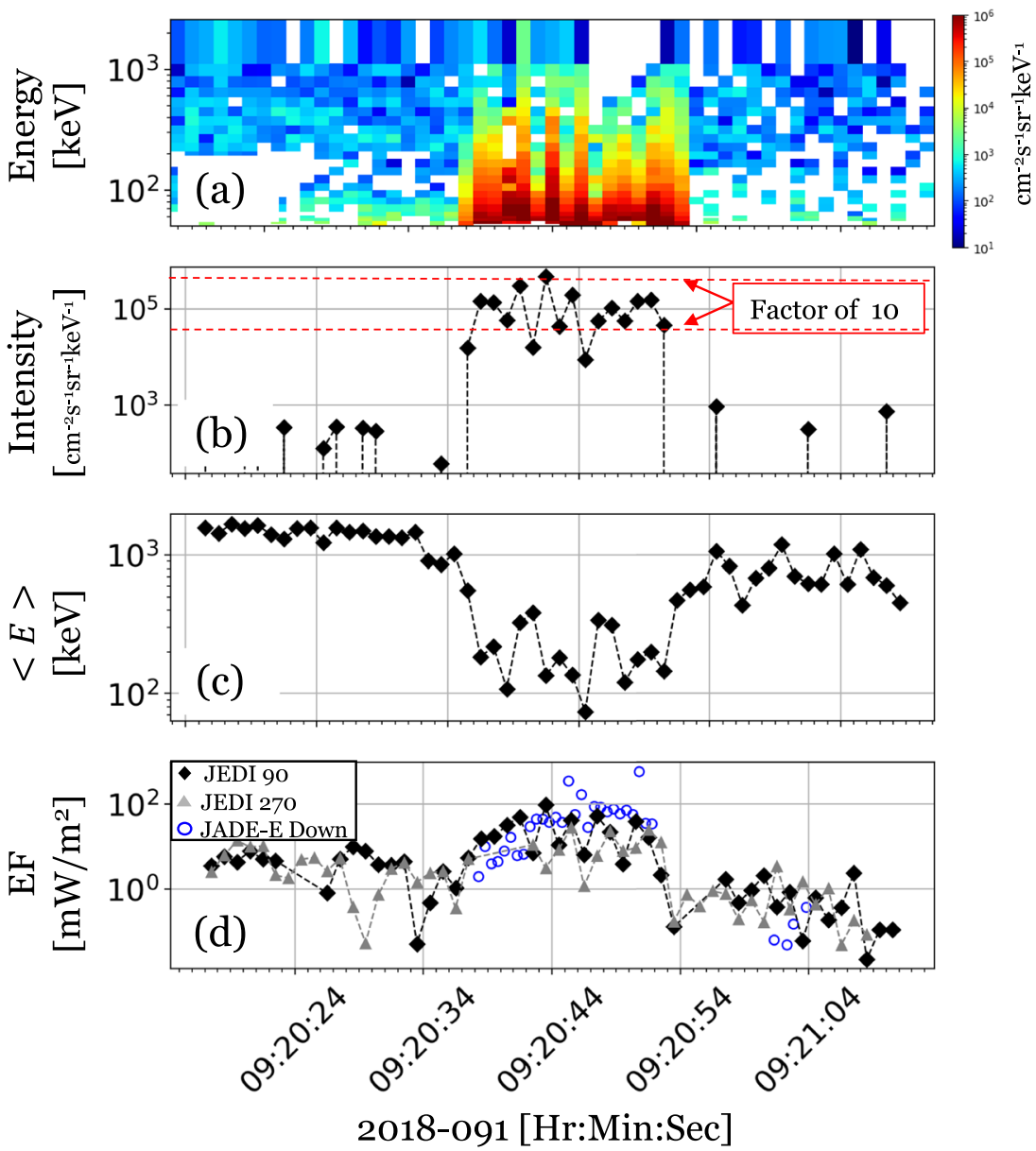

Figure 4. (a) JEDI J90 and J270 combined proton energy-time spectrogram filtered on pitch angles $40^{\circ}$ to $90^{\circ}$; (b) 100-keV proton intensities; (c) > 50-keV proton characteristic energies; (d) J90 (black diamonds), J270

(gray triangles) energetic proton energy flux versus JADE-E (blue circles) energy fluxes of plasma electrons $<40 \mathrm{keV}$. 
$\sim 100 \mathrm{~mW} / \mathrm{m}^{2}$ for the $\mathrm{J} 90$ sensor and $\sim 1$ and $\sim 30 \mathrm{~mW} / \mathrm{m}^{2}$ for the $\mathrm{J} 270$ sensor. Note that instantaneous pitch angle coverage is attributed to these differences. For comparison, we show the plasma electron (100 eV to $40 \mathrm{keV}$ ) precipitating energy fluxes (Szalay, Allegrini, et al., 2020) measured by Juno/JADE-E (McComas et al., 2017). JADE-E energy fluxes vary between $\sim 3$ and $600 \mathrm{~mW} / \mathrm{m}^{2}$.

\section{Discussion and Conclusions}

The angular distribution of energetic protons along the upward loss cone reveal strong evidence for energetic ion conic acceleration associated with IFP tail and probably the MAW. Ion conics are the result of thermal ionospheric ions heated perpendicular to the magnetic field via wave-particle interactions and then accelerated upward due to gradients in the magnetic field and/or field-aligned electric fields (e.g., Carlson et al., 1998; Chang, 1993; Gorney et al., 1985; Klumpar, 1979; Lynch et al., 2002; Retterer et al., 1994). Wave heating alone does not produce the most energetic ions; therefore, it is thought that electrostatic confinement via magnetic field-aligned potentials is required to trap the ions and further accelerate in the wave-heating region. This is referred to as the so-called "pressure cooker" mechanism (e.g., Gorney et al., 1985). Observations from Parker Solar Probe close to the Sun (Mitchell et al., 2020), Cassini at Saturn (Mitchell et al., 2009), and Juno at Jupiter (Clark, Mauk, Paranicas, et al., 2017) have confirmed their existence elsewhere in the solar system but have not been directly observed as a result of planet-moon interactions. Below, we discuss possible proton acceleration mechanisms associated with the IFP tail.

The first mechanism we consider is a cyclotron resonant heating mechanism. Sulaiman et al. (2020) analyzed the Juno/Waves measurements during the PJ12 IFP tail crossing and found evidence of upward-propagating, left-hand polarized ion cyclotron waves with large spectral densities (maximum of $\sim 10^{5} \mathrm{~V}^{2} / \mathrm{m}^{2} / \mathrm{Hz}$ ) near and at the proton cyclotron frequency. Using the theoretical energy transfer relationship from Chang et al. (1986), Sulaiman et al. (2020) estimated the ion heating rate, denoted as $d W_{\perp} / d t$, to have an upper limit of $\sim 500 \mathrm{eV} / \mathrm{s}$. To estimate the proton energies achievable from this heating rate, we need to know the time-of-flight of the ions between their source region and the spacecraft. First, the altitude of the source region can be estimated from the measured pitch angle distributions, shown in Figure 3, and by assuming the first adiabatic invariant is conserved as the protons are transported along the magnetic field. We also assume the protons are heated purely perpendicular to the local magnetic field, that is, pitch angles of $90^{\circ}$, in the source region (see similar method outlined in Clark, Mauk, Paranicas, et al., 2017 and references therein) and neglect changes in an ion's pitch angle as it is transported along the field line. The measured local magnetic field during the IFP tail crossing is $\sim 3 \times 10^{5} \mathrm{nT}$ and the centroid of the proton pitch angle distributions vary between $\sim 50^{\circ}$ and $60^{\circ}$. Combining this information together and using the latest magnetic field model (JRM09; Connerney et al., 2018), we find the source location to be $\sim 11,000 \mathrm{~km}$ or $0.16 R_{\mathrm{J}}$ above Jupiter's 1-bar oblate surface. The last piece of information required is the bulk velocity of ions in Jupiter's ionosphere. The only published ion bulk flow measurements in this region to date are from a study of low-energy ions in Jupiter's topside ionosphere using the JADE-Ion sensor (Valek et al., 2020). The authors performed a numerical integration of the plasma proton distributions and derived an outflow speed, $v_{b u l k}$, of $20 \mathrm{~km} / \mathrm{s}$. Here, we assume this to be the outflow speed of the protons in the region connected to the IFP tail, and thus, the time-of-flight of the protons is estimated to be approximately $t \approx 900 \mathrm{~s}$ where, $t=d / v_{\text {bulk }}$, where $d=18,500 \mathrm{~km}$ is the integrated length along the field between Juno at $0.33 R_{\mathrm{J}}$ and the source region at $0.16 R_{\mathrm{J}}$. Multiplying the proton's time-of-flight with the heating rate derived by Sulaiman et al. (2020) suggests ion cyclotron heating may be able to produce conic energies as large as $450 \mathrm{keV}$. This number is commensurate with the characteristic energies of the proton observations in the IFP tail (see Figure 4c). Major limitations of this crude estimate include the assumption that the wave heating is constant along the flux tube between the source region and the spacecraft and the bulk ion speed remains the same. While the wave-heating assumption appears to be reasonable in Earth's auroral region (e.g., Lynch et al., 2002), it is uncertain if the same holds true for Jupiter. Next, we consider the role of Alfvén waves as a possible energization mechanism.

The second mechanism we consider is Alfvénic acceleration. One striking observation can be found in Figure $4 \mathrm{~d}$ that shows the energetic ion energy flux as being comparable to the 0.1 - to $40-\mathrm{keV}$ electron energy flux, suggesting the energy partitioning between the two charged particle populations is similar. To estimate a power conversion efficiency, we use observations from magnetic field data. Gershman et al. (2019) analyzed the magnetic field fluctuations in Jupiter's polar magnetosphere and found direct evidence of strong 
transverse perturbations associated with the IFP tail. The perturbations were identified as Alfvénic (between 0.2 and $5 \mathrm{~Hz}$ ) and the Alfvén Poynting flux was calculated to be as high as $\sim 3,000 \mathrm{~mW} / \mathrm{m}^{2}$ during the likely PJ12 crossing of Io's MAW. Sulaiman et al. (2020) used Juno/Waves data to demonstrate that Alfvénic fluctuations, first observed by MAG, extend into the higher frequencies spanning a range from $\sim 50$ to $800 \mathrm{~Hz}$. Clearly, Alfvén waves are present and carry a significant source of energy in the IFP tail and near the MAW. Therefore, if the energy reservoir is the same for the two populations, then the observations presented here suggests that energy conversion efficiencies between Alfvén waves and ions ( 3-5\%) are comparable to the lower-energy electrons except for brief moments where the electron energy flux peaks as high as $580 \mathrm{~mW} / \mathrm{m}^{2}$. This is surprising because previous works, for example, Hess et al. (2010), demonstrate that most of the Alfvénic Poynting flux is converted to electrons. We note that numerous studies have investigated ion acceleration in Earth's aurorae and the role of Alfvén waves (e.g., Chaston et al., 2004, 2015; Johnson \& Cheng, 2001; Knudsen \& Wahlund, 1998; Li \& Temerin, 1993; White et al., 2002). However, in the absence of wave-particle interaction models for the Io fluxtube and its tail, which consider the ion response specifically, we turn to comparisons with models at larger L-shells. Saur et al. (2018) find that on L-shells between 10 and $40 R_{\mathrm{J}}$, at high latitudes, ion-Landau damping is effectively not taking place, while electron Landau damping of inertial Alfvén waves is a highly effective acceleration mechanism in accordance with previous modeling and existing observations of energetic electrons (e.g., Bonfond et al., 2017; Clark et al., 2018; Hess et al., 2010, 2013; Saur et al., 2018; Szalay, Allegrini, et al., 2020). If the temporal scales of the waves become extremely small, then ion cyclotron damping becomes more prominent (e.g., Sulaiman et al., 2020). Of the two resonant mechanisms discussed, that is, Landau and cyclotron damping, non-resonant mechanisms (e.g., Lu \& Li, 2007) of ion acceleration through Alfvén waves have not been studied for the Jupiter system and their effectiveness is thus difficult to assess without detailed studies.

The Juno/JEDI data presented in this study represent the first measurements of energetic proton conics associated with IFP tail near the MAW. This discovery showcases the diversity of planetary systems and interactions present where ion conics exist, for example, Earth, Saturn, and Jupiter's auroral regions and now as a result of moon-magnetospheric interactions. Our primary conclusions in this study are the following:

1. Energetic proton acceleration associated with the IFP tail appears significant and perhaps the most intense ion event recorded by Juno/JEDI to date.

2. The angular distributions of the protons suggest these are the ion conic distributions and are likely accelerated by ion cyclotron waves via a resonant interaction; however, Alfvénic turbulence was not ruled out and may play a role.

3. Proton acceleration associated with IFP tail is more intense than compared to the main auroral (Mauk et al., 2018) or polar cap regions (Clark, Mauk, Paranicas, et al., 2017), thus highlighting the unique and strong electromagnetic interaction between Jupiter and Io.

\section{Data Availability Statement}

All Juno data presented here are publicly available from NASA's Planetary Data System as part of the JNO-JJED-3_CDR-V1.0 data set for the Juno/JEDI instrument and The JNO-J/SW-JAD-3-CALIBRATED-V2.0 and JNO-J/SW-JAD-2-UNCALIBRATED-V1.0 for the Juno/JADE instrument.

\section{Acknowledgments}

The authors would like to thank Don Mitchell and Matina Gkioulidou for their discussions and assistance in understanding the corrected rates of the instrument. This work was supported by the Juno mission. The research at the University of Iowa was supported by NASA through Contract $699041 X$ with the Southwest Research Institute.

\section{References}

Acuña, M. H., Neubauer, F. M., \& Ness, N. F. (1981). Standing Alfvén wave current system at Io: Voyager 1 observations. Journal of Geophysical Research, 86(A10), 8513-8521. https://doi.org/10.1029/JA086iA10p08513

Bagenal, F. (1983). Alfvén wave propagation in the Io plasma torus. Journal of Geophysical Research, 88(A4), 3013-3025. https://doi.org/ 10.1029/JA088iA04p03013

Bagenal, F., Adriani, A., Allegrini, F., Bolton, S. J., Bonfond, B., Bunce, E. J., et al. (2017). Magnetospheric science objectives of the Juno mission. Space Science Reviews, 213(1-4), 219-287. https://doi.org/10.1007/s11214-014-0036-8

Belcher, J. W., Goertz, C. K., Sullivan, J. D., \& Acuña, M. H. (1981). Plasma observations of the Alfvén wave generated by Io. Journal of Geophysical Research, 86(A10), 8508-8512. https://doi.org/10.1029/JA086iA10p08508

Bigg, E. K. (1964). Influence of the satellite Io on Jupiter's decametric emission. Nature, 203(4949), 1008-1010. https://doi.org/10.1038/ $2031008 \mathrm{a} 0$

Bonfond, B., Grodent, D., Gérard, J. C., Radioti, A., Dols, V., Delamere, P. A., \& Clarke, J. T. (2009). The Io UV footprint: Location, inter-spot distances and tail vertical extent. Journal of Geophysical Research, 114, A07224. https://doi.org/10.1029/2009JA014312

Bonfond, B., Grodent, D., Gérard, J.-C., Radioti, A., Saur, J., \& Jacobsen, S. (2008). UV Io footprint leading spot: A key feature for understanding the UV Io footprint multiplicity? Geophysical Research Letters, 35, L05107. https://doi.org/10.1029/2007GL032418 
Bonfond, B., Hess, S., Gérard, J. C., Grodent, D., Radioti, A., Chantry, V., et al. (2013). Evolution of the Io footprint brightness I: Far-UV observations. Planetary and Space Science, 88, 64-75. https://doi.org/10.1016/j.pss.2013.05.023

Bonfond, B., Saur, J., Grodent, D., Badman, S. V., Bisikalo, D., Shematovich, V., et al. (2017). The tails of the satellite auroral footprints at Jupiter. Journal of Geophysical Research: Space Physics, 122, 7985-7996. https://doi.org/10.1002/2017JA024370

Carlson, C. W., McFadden, J. P., Ergun, R. E., Temerin, M., Peria, W., Mozer, F. S., et al. (1998). FAST observations in the downward auroral current region: Energetic upgoing electron beams, parallel potential drops, and ion heating. Geophysical Research Letters, 25(12), 2017-2020. https://doi.org/10.1029/98GL00851

Chang, T. (1993). Lower-hybrid collapse, caviton turbulence, and charged particle energization in the topside auroral ionosphere and magnetosphere*. Physics of Fluids B: Plasma Physics, 5(7), 2646-2656. https://doi.org/10.1063/1.860702

Chang, T., Crew, G. B., Hershkowitz, N., Jasperse, J. R., Retterer, J. M., \& Winningham, J. D. (1986). Transverse acceleration of oxygen ions by electromagnetic ion cyclotron resonance with broad band left-hand polarized waves. Geophysical Research Letters, 13(7), 636-639. https://doi.org/10.1029/GL013i007p00636

Chaston, C. C., Bonnell, J. W., Carlson, C. W., McFadden, J. P., Ergun, R. E., Strangeway, R. J., \& Lund, E. J. (2004). Auroral ion acceleration in dispersive Alfvén waves. Journal of Geophysical Research, 109, A04205. https://doi.org/10.1029/2003JA010053

Chaston, C. C., Bonnell, J. W., Wygant, J. R., Kletzing, C. A., Reeves, G. D., Gerrard, A., et al. (2015). Extreme ionospheric ion energization and electron heating in Alfvén waves in the storm time inner magnetosphere. Geophysical Research Letters, 42, 10,531-10,540. https:// doi.org/10.1002/2015GL066674

Clark, G., Mauk, B. H., Haggerty, D., Paranicas, C., Kollmann, P., Rymer, A., et al. (2017). Energetic particle signatures of magnetic field-aligned potentials over Jupiter's polar regions. Geophysical Research Letters, 44, 8703-8711. https://doi.org/10.1002/2017GL074366

Clark, G., Mauk, B. H., Paranicas, C., Haggerty, D., Kollmann, P., Rymer, A., et al. (2017). Observation and interpretation of energetic ion conics in Jupiter's polar magnetosphere. Geophysical Research Letters, 44, 4419-4425. https://doi.org/10.1002/2016GL072325

Clark, G., Tao, C., Mauk, B. H., Nichols, J., Saur, J., Bunce, E. J., et al. (2018). Precipitating electron energy flux and characteristic energies in Jupiter's main auroral region as measured by Juno/JEDI. Journal of Geophysical Research: Space Physics, 123, 7554-7567. https://doi. org/10.1029/2018JA025639

Clarke, J. T., Ballester, G. E., Trauger, J., Evans, R., Connerney, J. E. P., Stapelfeldt, K., et al. (1996). Far-ultraviolet imaging of Jupiter's aurora and the Io "footprint". Science, 274(5286), 404-409. https://doi.org/10.1126/science.274.5286.404

Connerney, J. E. P., Adriani, A., Allegrini, F., Bagenal, F., Bolton, S. J., Bonfond, B., et al. (2017). Jupiter's magnetosphere and aurorae observed by the Juno spacecraft during its first polar orbits. Science, 356, 826-832. https://doi.org/10.1126/science.aam5928

Connerney, J. E. P., Baron, R., Satoh, T., \& Owen, T. (1993). Images of excited $\mathrm{H}_{3}{ }^{+}$at the foot of the lo flux tube in Jupiter's atmosphere. Science, 262(5136), 1035-1038. https://doi.org/10.1126/science.262.5136.1035

Connerney, J. E. P., Benn, M., Bjarno, J. B., Denver, T., Espley, J., Jorgensen, J. L., et al. (2017). The Juno magnetic field investigation. Space Science Reviews, 213, 39-138. https://doi.org/10.1007/s11214-017-0334-z

Connerney, J. E. P., Kotsiaros, S., Oliversen, R. J., Espley, J. R., Jørgensen, J. L., Joergensen, P. S., et al. (2018). A new model of Jupiter's magnetic field from Juno's first nine orbits. Geophysical Research Letters, 45, 2590-2596. https://doi.org/10.1002/2018GL077312

Crary, F. J., \& Bagenal, F. (1997). Coupling the plasma interaction at Io to Jupiter. Geophysical Research Letters, 24(17), 2135-2138. https:// doi.org/10.1029/97GL02248

Damiano, P. A., Delamere, P. A., Stauffer, B., Ng, C.-S., \& Johnson, J. R. (2019). Kinetic simulations of electron acceleration by dispersive scale Alfven waves in Jupiter's magnetosphere. Geophysical Research Letters, 46, 3043-3051. https://doi.org/10.1029/2018GL081219

Frank, L. A., Paterson, W. R., Ackerson, K. L., Vasyliunas, V. M., Coroniti, F. V., \& Bolton, S. J. (1996). Plasma observations at Io with the Galileo spacecraft. Science, 274(5286), 394-395. https://doi.org/10.1126/science.274.5286.394

Gerard, J.-C., Saglam, A., Grodent, D., \& Clarke, J. T. (2006). Morphology of the ultraviolet emission and its control by Io's location. Journal of Geophysical Research, 111, A04202. https://doi.org/10.1029/2005JA011327

Gershman, D. J., Connerney, J. E. P., Kotsiaros, S., DiBraccio, G. A., Martos, Y. M., Viñas, A. F., et al. (2019). Alfvénic fluctuations associated with Jupiter's auroral emissions. Geophysical Research Letters, 46, 7157-7165. https://doi.org/10.1029/2019GL082951

Gladstone, G. R., Persyn, S. C., Eterno, J. S., Walther, B. C., Slater, D. C., Davis, M. W., et al. (2017). The ultraviolet spectrograph on NASA's Juno mission. Space Science Reviews, 213, 447-473. https://doi.org/10.1007/s11214-014-0040-z

Goertz, C. (1980). Io's interaction with the plasma torus. Journal of Geophysical Research, 85(A6), 2949-2956. https://doi.org/10.1029/ JA085iA06p02949

Goldreich, P., \& Lynden-Bell, D. (1969). Io, a Jovian unipolar inductor. The Astrophysical Journal, 156, 59-78. https://doi.org/10.1086/ 149947

Gorney, D. J., Chiu, Y. T., \& Croley, D. R. Jr. (1985). Trapping of ion conics by downward parallel electric fields. Journal of Geophysical Research, 90(A5), 4205-4210. https://doi.org/10.1029/JA090iA05p04205

Grodent, D., Clarke, J. T., Kim, J., Waite, J. H. Jr., \& Cowley, S. W. H. (2003). Jupiter's main auroral oval observed with HST-STIS. Journal of Geophysical Research, 108(A11), 1389. https://doi.org/10.1029/2003JA009921

Gurnett, D. A., \& Goertz, C. K. (1981). Multiple Alfven wave reflections excited by Io: Origin of the Jovian decametric arcs. Journal of Geophysical Research, 86(A2), 717-722. https://doi.org/10.1029/JA086iA02p00717

Gurnett, D. A., Kurth, W. S., Roux, A., Bolton, S. J., \& Kennel, C. F. (1996). Galileo plasma wave observations in the Io plasma torus and near Io. Science, 274(5286), 391-392. https://doi.org/10.1126/science.274.5286.391

Hess, S. L., Delamere, P., Dols, V., Bonfond, B., \& Swift, D. (2010). Power transmission and particle acceleration along the Io flux tube. Journal of Geophysical Research, 115, A06205. https://doi.org/10.1029/2009JA014928

Hess, S. L. G., Bonfond, B., Chantry, V., Gérard, J. C., Grodent, D., Jacobsen, S., \& Radioti, A. (2013). Evolution of the Io footprint brightness II: Modeling. Planetary and Space Science, 88, 76-85. https://doi.org/10.1016/j.pss.2013.08.005

Hue, V., Greathouse, T. K., Bonfond, B., Saur, J., Gladstone, G. R., Roth, L., et al. (2019). Juno-UVS observation of the Io footprint during solar eclipse. Journal of Geophysical Research: Space Physics, 124, 5184-5199. https://doi.org/10.1029/2018JA026431

Johnson, J. R., \& Cheng, C. Z. (2001). Stochastic ion heating at the magnetopause due to kinetic Alfven waves. Geophysical Research Letters, 28(23), 4421-4424. https://doi.org/10.1029/2001GL013509

Jones, S. T., \& Su, Y.-J. (2008). Role of dispersive Alfvén waves in generating parallel electric fields along the Io-Jupiter fluxtube. Journal of Geophysical Research, 113, A12205. https://doi.org/10.1029/2008JA013512

Kivelson, M. G., Khurana, K. K., Walker, R. J., Russell, C. T., Linker, J. A., Southwood, D. J., \& Polanskey, C. (1996). A magnetic signature at Io: Initial report from the Galileo magnetometer. Science, 273(5273), 337-340. https://doi.org/10.1126/science.273.5273.337

Klumpar, D. M. (1979). Transversely accelerated ions: An ionospheric source of hot magnetospheric ions. Journal of Geophysical Research, 84(A8), 4229-4237. https://doi.org/10.1029/JA084iA08p04229 
Knudsen, D. J., \& Wahlund, J. E. (1998). Core ion flux bursts within solitary kinetic Alfvén waves. Journal of Geophysical Research, 103(A3), 4157-4169. https://doi.org/10.1029/97JA01679

Kurth, W. S., Hospodarsky, G. B., Kirchner, D. L., Mokrzycki, B. T., Averkamp, T. F., Robison, W. T., et al. (2017). The Juno waves investigation. Space Science Reviews, 213, 347-392. https://doi.org/10.1007/s11214-017-0396-y

Li, X., \& Temerin, M. (1993). Ponderomotive effects on ion acceleration in the auroral zone. Geophysical Research Letters, 20(1), 13-16. https://doi.org/10.1029/92GL03011

Lu, Q., \& Li, X. (2007). Heating of ions by low-frequency Alfven waves. Physics of Plasmas, 14, 042303. https://doi.org/10.1063/1.2715569

Lynch, K. A., Bonnell, J. W., Carlson, C. W., \& Peria, W. J. (2002). Return current region aurora: $\mathrm{E}_{\|}, \mathrm{j}_{z}$, particle energization, and broadband ELF wave activity. Journal of Geophysical Research, 107(A7), 1115. https://doi.org/10.1029/2001JA900134

Mauk, B. H., Haggerty, D. K., Jaskulek, S. E., Schlemm, C. E., Brown, L. E., Cooper, S. A., et al. (2017). The Jupiter Energetic Particle Detector Instrument (JEDI) investigation for the Juno Mission. Space Science Reviews, 213, 289-346. https://doi.org/10.1007/s11214-0130025-3

Mauk, B. H., Haggerty, D. K., Paranicas, C., Clark, G., Kollmann, P., Rymer, A. M., et al. (2017). Discrete and broadband electron acceleration in Jupiter's powerful aurora. Nature, 549, 66-69. https://doi.org/10.1038/nature23648

Mauk, B. H., Haggerty, D. K., Paranicas, C. P., Clark, G., Kollmann, P., Rymer, A. M., et al. (2018). Diverse electron and ion acceleration characteristics observed over Jupiter's main aurora. Geophysical Research Letters, 45, 1277-1285. https://doi.org/10.1002/2017GL076901

Mauk, B. H., Mitchell, D. G., McEntire, R. W., Paranicas, C. P., Roelof, E. C., Williams, D. J., et al. (2004). Energetic ion characteristics and neutral gas interactions in Jupiter's magnetosphere. Journal of Geophysical Research, 109, A09S12. https://doi.org/10.1029/ 2003JA010270

McComas, D. J., Alexander, N., Allegrini, F., Bagenal, F., Beebe, C., Clark, G., et al. (2017). The Jovian Auroral Distributions Experiment (JADE) on the Juno Mission to Jupiter. Space Science Reviews, 213, 547-643. https://doi.org/10.1007/s11214-013-9990-9

Mitchell, D. G., Giacalone, J., Allen, R. C., Hill, M. E., McNutt, R. L., McComas, D. J., et al. (2020). CME-associated energetic ions at 0.23 au: Consideration of the auroral pressure cooker mechanism operating in the low corona as a possible energization process. The Astrophysical Journal Supplement Series, 246, 59. http://doi.org/10.3847/1538-4365/ab63cc

Mitchell, D. G., Kurth, W. S., Hospodarsky, G. B., Krupp, N., Saur, J., Mauk, B. H., et al. (2009). Ion conics and electron beams associated with auroral processes on Saturn. Journal of Geophysical Research, 114, A02212. https://doi.org/10.1029/2008JA013621

Neubauer, F. (1980). Nonlinear standing Alfvén wave current system at Io: Theory. Journal of Geophysical Research, 85(A3), 1171-1178. https://doi.org/10.1029/JA085iA03p01171

Paranicas, C., Mauk, B. H., Haggerty, D. K., Clark, G., Kollmann, P., Rymer, A. M., et al. (2018). Intervals of intense energetic electron beams over Jupiter's poles. Journal of Geophysical Research: Space Physics, 123, 1989-1999. https://doi.org/10.1002/2017JA025106

Prangé, R., Rego, D., Southwood, D., Zarka, P., Miller, S., \& Ip, W. (1996). Rapid energy dissipation and variability of the lo-Jupiter electrodynamic circuit. Nature, 379(6563), 323-325. https://doi.org/10.1038/379323a0

Queinnec, J., \& Zarka, P. (1998). Io-controlled decameter arcs and Io-Jupiter interaction. Journal of Geophysical Research, 103(A11), 26,649-26,666. https://doi.org/10.1029/98JA02435

Retterer, J. M., Chang, T., \& Jasperse, J. R. (1994). Transversely accelerated ions in the topside ionosphere. Journal of Geophysical Research, 99(A7), 13,189-13,201. https://doi.org/10.1029/93JA03570

Saur, J. (2004). A model of Io's local electric field for a combined Alfvénic and unipolar inductor far-field coupling. Journal of Geophysical Research, 109, A01210. https://doi.org/10.1029/2002JA009354

Saur, J., Grambusch, T., Duling, S., Neubauer, F. M., \& Simon, S. (2013). Magnetic energy fluxes in sub-Alfvénic planet star and moon planet interactions. A\&A, 552, A119. Retrieved from. https://doi.org/10.1051/0004-6361/201118179

Saur, J., Janser, S., Schreiner, A., Clark, G., Mauk, B. H., Kollmann, P., et al. (2018). Wave-particle interaction of Alfvén waves in Jupiter's magnetosphere: Auroral and magnetospheric particle acceleration. Journal of Geophysical Research: Space Physics, 123, $9560-9573$. https://doi.org/10.1029/2018JA025948

Sulaiman, A. H., Hospodarsky, G. B., Elliott, S. S., Kurth, W. S., Gurnett, D. A., Imai, M., et al. (2020). Wave-particle interactions associated with Io's auroral footprint: Evidence of Alfvén, ion cyclotron, and whistler modes. Geophysical Research Letters, 47, e2020GL088432. https://doi.org/10.1029/2020GL088432

Szalay, J. R., Allegrini, F., Bagenal, F., Bolton, S. J., Bonfond, B., Clark, G., et al. (2020). A new framework to explain changes in Io's footprint tail electron fluxes. Geophysical Research Letters, 47, e2020GL089267. https://doi.org/10.1029/2020GL089267

Szalay, J. R., Bagenal, F., Allegrini, F., Bonfond, B., Clark, G., Connerney, J. E. P., et al. (2020). Proton acceleration by Io's Alfvénic interaction. Journal of Geophysical Research: Space Physics, 125, e2019JA027314. https://doi.org/10.1029/2019JA027314

Szalay, J. R., Bonfond, B., Allegrini, F., Bagenal, F., Bolton, S., Clark, G., et al. (2018). In situ observations connected to the Io footprint tail aurora. Journal of Geophysical Research: Planets, 123, 3061-3077. https://doi.org/10.1029/2018JE005752

Valek, P. W., Bagenal, F., Ebert, R. W., Allegrini, F., McComas, D. J., Szalay, J. R., et al. (2020). Juno in situ observations above the Jovian equatorial ionosphere. Geophysical Research Letters, 47, e2020GL087623. https://doi.org/10.1029/2020GL087623

White, R., Chen, L., \& Lin, Z. (2002). Resonant plasma heating below the cyclotron frequency. Physics of Plasmas, 9(5), 1890-1897. https:// doi.org/10.1063/1.1445180

Williams, D. J., Mauk, B. H., McEntire, R. E., Roelof, E. C., Armstrong, T. P., Wilken, B., et al. (1996). Electron beams and ion composition measured at Io and in its torus. Science, 274(5286), 401-403. https://doi.org/10.1126/science.274.5286.401

Zarka, P. (2000). Radio emissions from the planets and their moons. Geophysical Monograph-American Geophysical Union, 119, 167-178. 